\title{
Successful Experimental Infant Baboon Model for Childhood Cryptosporidiosis Studies
}

Ngalla Edward Jillani ( $\square$ ngalla.jillani@gmail.com )

Institute of Primate Research https://orcid.org/0000-0002-0353-8514

Atunga Nyachieo

IPR: Institute of Primate Research

Chivatsi Daniel Chai

IPR: Institute of Primate Research

Nyabuga James Nyariki

TU-K: Technical University of Kenya

\section{Research Article}

Keywords: Cryptosporidiosis, infant baboon, Cryptosporidium parvum, children

Posted Date: June 3rd, 2021

DOI: https://doi.org/10.21203/rs.3.rs-268825/v1

License: (c) (1) This work is licensed under a Creative Commons Attribution 4.0 International License. Read Full License 


\section{Abstract}

Background: Cryptosporidiosis causes high morbidity and fatality to children under two years globally. The lack of an appropriate animal model that mimics the pathogenesis of disease in humans has hampered the development and testing of potential therapeutic options. This study aimed to develop and validate an infant baboon infection model of cryptosporidiosis.

Methods: Eighteen immunocompetent weaned infant baboons aged 12 to 16 months were used. The animals were: $n=3$ controls and three experimental groups of $n=5$ animals each inoculated with Cryptosporidium parvum oocysts as follows: Group 1: $2 \times 10^{4}$, Group 2: $2 \times 10^{5}$, Group 3: $2 \times 10^{6}$ followed by daily fecal sampling for oocysts evaluation. Blood sampling for immunological assay was done on infection day and thereafter weekly until the end of the experiment followed by necropsy and histopathology. Statistical analysis was done using R, statistical package for the social sciences (SPSS) and Graph Pad Prism software. Analysis of variance (ANOVA) and Bonferroni post-hoc tests were used for comparison of the means, with $p<0.05$ considered as a significant difference. Correlation coefficient and Probit analysis were also performed.

Results: In all experimental animals but not controls, the onset of oocysts shedding occurred between days 2 and 4, with the highest oocyst shedding occurring between days 6 and 28. Histological analysis revealed parasites establishment only in infected animals. Levels of cytokines (TNF-a, IFN- $y$, and IL-10) significantly increased in experimental groups compared to controls.

Conclusion: $2 \times 10^{4}$ oocysts were an effective minimum quantifiable experimental infection dose for developing a reproducible infant baboon model.

\section{Background}

Cryptosporidiosis is an infectious disease caused by an apicomplexan oocyst-forming protozoan parasite, Cryptosporidium spp. [1, 2]. Besides the two main species of Cryptosporidium infecting humans, namely, $C$. hominis (which infects humans only) and $C$. parvum (which infects both humans and bovines), other species such as $C$. suis, $C$. meleagridis, $C$. andersoni, $C$. felis, $C$. canis, $C$. muris, and $C$. baylei, occasionally infect people as well $[2,3]$. This enteropathogenic disease is associated with diarrhea in humans of all ages, but more severe in children under two years worldwide [4, 5]. This neglected zoonotic disease, with a usually underestimated prevalence of $2-12 \%$ in the developing world, has received little research attention leading to limited understanding of its pathogenesis and less effective therapeutic solutions $[3,6]$. Childhood cryptosporidiosis, presents a huge disease burden in the developing world, causing morbidity and fatality with possible long term learning deficits, stunted growth, and impaired physical fitness in affected children $[2,6-8]$.

Transmission is by the fecal-oral route through direct contact of infected humans, animals, contaminated drinking water, or agricultural products [9]. Oocysts excreted in feces can remain infectious for up to two months [10]. This disease causes opportunistic infections in immune-competent as well as immune- 
compromised persons [5]. While incidences of cryptosporidiosis vary throughout the year, they become high during the warm rainy months [8].

Despite numerous attempts to control Cryptosporidium infections, there have not been effective animal models that accurately mimic the disease in humans, as tools for testing newly developed or repurposed molecules for potential drugs that could effectively treat this condition $[2,7]$. To better understand the disease, various animal models, mainly murine and porcine models have been utilized [11]. The findings from these lower animal models, however, cannot be extrapolated to humans since the disease pattern does not mirror that in humans due to the huge phylogenetic gap. Non-human primates, which are phylogenetically closer to humans are, therefore, attractive as models for the understanding of this disease [7]. Indeed, previous studies in pigtailed macaques demonstrated that these non-human primates were good models of the disease [7]. Similarly, in another study involving a cross-sectional survey in captive baboons [12], C. hominis infection was detected, mostly, in infant baboons confirming that baboon infants are susceptible to Cryptosporidium hominis infection and could be useful in the development of a reproducible experimental infection model of childhood cryptosporidiosis. However, there is no study showing dose-controlled Cryptosporidium infection in baboon infants. Thus, the main objective of this study was to validate and develop a reproducible infant baboon infection model of childhood cryptosporidiosis through a quantifiable Cryptosporidium dosage using Cryptosporidium parvum.

\section{Materials And Methods}

\section{Selection of animals}

Weaned immunocompetent infant baboons $(n=18)$ of both sexes ( 10 males and 8 females) aged between 12 -16 months, and weighing between 3.0 - 7.1 Kgs were recruited into the study. The sample size (n) was calculated using the power analysis equation; $n=1+2 C(s / d)^{2}$. The animals were screened, treated for enteric worms, and diarrhea-causing bacteria, namely Shigella spp. and Salmonella spp. before inoculation with doses of Cryptosporidium parvum oocysts. They were then housed in cages that meet international standards in sizes and space based on the NIH guide on care and use of laboratory animals, $8^{\text {th }}$ edition. Animals were fed on commercial chow made by Unga Feeds Ltd, based on our formulation, while water was given ad libitum. They were given fruits thrice a week and provided with enrichment gadgets, which were changed weekly.

\section{Isolation of Cryptosporidium oocysts}

Cryptosporidium parvum oocysts used in this study were isolated by the floatation method and identified by acid-fast staining (modified Ziehl-Neelsen method) as previously described [7]. In this method, a lump of the fecal sample (about $1 \mathrm{~g}$ ) was put into a container and $5 \mathrm{ml}$ of distilled water added, thoroughly mixed then sieved into a beaker. The filtrate was thereafter transferred into a $15 \mathrm{ml}$ centrifuge tube and topped up with distilled water to $15 \mathrm{ml}$ then centrifuged at $1500 \mathrm{rpm}$ for 10 minutes. The supernatant was 
discarded and $5 \mathrm{ml}$ of sucrose solution of specific gravity 1.27 added and vortexed for 1 minute. More sucrose solution was added to make it to $15 \mathrm{ml}$, until a convex meniscus was formed, covered with a coverslip and centrifuge at 1500rpm for 10 minutes. The coverslip was then carefully removed and washed by sprinkling PBS on it into a glass beaker. The $15 \mathrm{ml}$ tube was again topped up with sucrose solution till it formed a meniscus, covered with a coverslip, centrifuged for another 10 minutes at 1500rpm, and oocysts recovered as described earlier. This process was repeated three times to ensure the maximum recovery of oocysts. The resultant solution with recovered oocysts from the beaker was then put into a $15 \mathrm{ml}$ tube, topped up with PBS, and centrifuge at 1500rpm for 10 minutes. The supernatant was discarded and the resultant pellets were suspended in $1.5 \mathrm{ml} \mathrm{PBS}$. One microliter $(1 \mu \mathrm{l})$ of this solution was examined under a microscope to determine the number of oocysts in $1 \mu \mathrm{l}$ volume. This volume estimation for oocyst numbers was then used to constitute appropriate inoculation doses.

\section{Inoculation of animals}

All selected experimental animals $(n=18)$ were starved in the morning of inoculation and the procedures carried out under general anesthesia. This involved injecting Ketamine $\mathrm{HCl}$ at $10 \mathrm{mgs} / \mathrm{kg}$ body weight mixed with xylazine $5 \%$, intramuscular. During this time, blood samples, temperatures, and body weights were taken. The animals were randomized to three experimental groups using sealed opaque envelopes. Quantified parasite doses were administered to the animals on the table according to the groups. The

doses comprised of three concentrations of the parasite; Group 1: $2 \times 10^{4}(n=5)$, Group 2: $2 \times 10^{5}(n=5)$, and Group 3: $2 \times 10^{6}(n=5)$. Group 4 comprised of control animals $(n=3)$ were given normal saline. Parasite dose administration was done through a thin tube pushed into the oral-gastric route and the parasite's oocysts delivered through a syringe connected to the tube and washed several times into the gastrointestinal tract. The animals were then returned to their cages and closely monitored till they recovered from the effects of anesthesia.

\section{Stool sampling and oocysts quantification}

Stool sampling for quantification of shed oocysts was done daily by placing a tray at the base of the cage, just below the animal. Part of freshly voided fecal matter was put in sealable vials and stored at four degrees $\left(4^{\circ} \mathrm{C}\right)$. Later, $100 \mathrm{gms}$ of each sampled stool were concentrated by the formalin- ethyl acetate sedimentation method and stained using the modified Ziehl-Neelsen method [7]. A 5 $\mu$ l volume of the isolated oocysts was sampled, counted under a microscope $(x 400)$ and total numbers recorded.

\section{DNA extraction from oocysts}

Chromosomal DNA was extracted by QIA amp DNA kit (Qiagen, Hilden, Germany) according to the manufacturer's instructions. $5 \mu \mathrm{l}$ of pure oocysts was transferred into an Eppendorf tube and dissolved in $700 \mu \mathrm{L}$ of ASL buffer of the DNA extraction kit. This was then exposed to five cycles of freeze and thaw within liquid nitrogen and boiling water to disrupt oocyst walls. Afterward, $700 \mu \mathrm{L}$ of ASL buffer was added into the sample tube and then the procedure, based on the instruction of the DNA extraction kit was followed. 


\section{Nested PCR amplification}

PCR was carried out using a peqSTAR thermocycler (Peqlab, Erlangen, Germany) as described previously [13]. Briefly, 18S ribosomal RNA gene from Cryptosporidium genus (NCBI, Accession No. GQ259149.1) was amplified under the following conditions for analysis: $94^{\circ} \mathrm{C}$ for $5 \mathrm{~min}, 35$ cycles of 1 min at $94{ }^{\circ} \mathrm{C}$, $1.30 \mathrm{~min}$ at $60^{\circ} \mathrm{C}, 2 \mathrm{~min}$ at $72^{\circ} \mathrm{C}$ (Cry18S-S2, 5'GGTGACTCATAATAACTTTACGG 3' as forward and Cry18S-As2, 5'ACGCTATTGGAGCTGGAATTAC $3^{\prime}$ as reverse primers). This was followed by nested amplification of 35 cycles: $1 \mathrm{~min}$ at $94^{\circ} \mathrm{C}, 1.30 \mathrm{~min}$ at $60^{\circ} \mathrm{C}, 2 \mathrm{~min}$ at $72{ }^{\circ} \mathrm{C}$ and a final extension step of 10 min at $72{ }^{\circ} \mathrm{C}$ (Cry18S-S1, 5'TAAACGGTAGGGTATTGGCCT 3' as forward and Cry18S-As1, 5' CAGACTTGCCCTCCAATTGATA3' as the reverse. Twenty (20) $\mathrm{pM}$ of each primer was added in a volume of $50 \mu$ l containing: $20 \mathrm{mM}(\mathrm{NH} 4) 2 \mathrm{SO} 4,75 \mathrm{mM}$ Tris-HCl (pH. 8.8), $1 \mathrm{mM} \mathrm{MgCl} 2,0.2 \mathrm{mM}$ dNTP mix, 1.2 Units of thermostable DNA polymerase (Invitrogen, Mannheim, Germany), and $1 \mu \mathrm{l}$ of the template (genomic DNA). The amplification products were subjected to electrophoresis on a $1 \%$ agarose gel stained with ethidium bromide and visualized under a UV transilluminator. The expected PCR band size after nested PCR was approximately 240bp.

\section{Sequencing}

The PCR bands were extracted from the agarose gel and purified then sequenced by Macrogen Co. Ltd. (Netherlands). The sequenced PCR fragment was then blasted at NCBI (https://blast.ncbi.nlm.nih.gov/Blast.cgi) to identify the sequenced gene.

\section{Blood sampling}

Six (6) $\mathrm{ml}$ of whole blood was collected from each animal on inoculation day and seven days thereafter for processing blood cell count and cytokine assays. Blood was collected from femoral vein under anesthesia attained by intramuscular injection of Ketamine-xylazine mixture with Ketamine $\mathrm{HCl}$ at a dosage of $10 \mathrm{mgs} / \mathrm{kg}$ body weight, and xylazine $5 \%$. While $3 \mathrm{ml}$ of the collected blood was processed for cell count, the other $3 \mathrm{ml}$ were processed for serum, which was then stored at minus twenty degrees Celsius $\left(-20^{\circ} \mathrm{C}\right)$. Cell counting was done using an automated differential cell counter (Coulter Ac.T 5 diff CP, Beckman Coulter, Miami, USA). Serum was separated from whole blood through centrifugation using a Jouan centrifuge (Jouan, C422, LabCare America, Florida, USA) at $1500 \mathrm{xg}$ for ten (10) minutes and then collected into a clean tube for storage.

\section{Temperature and body weight}

Body temperatures were recorded using a rectal thermometer (Hongwei Technology Co. Ltd, Hong Kong) inserted into the anal opening, while body weights were taken on a weighing balance. These were taken during the day of inoculation and weekly, thereafter, for the duration of the experiment.

\section{Immunological analysis}


From the serum of the collected blood samples, inflammatory cytokines TNF-a, IFN- $\gamma$, and IL-10 were analyzed using ELISA kits (Human TNF-a kit, IFN- $\gamma$ kit, and IL-10 kit; Thermofisher Scientific Ltd, Vienna, Austria) at acute phase day 14 and chronic phase day 49 for both control and experimental animals. Sandwich ELISA protocol was employed for the detection of the presence and levels of the inflammatory cytokines TNF-a, IFN- $y$, and IL-10. Briefly, Corning Costar 9018 low bottom high binding ELISA plates (Thermofisher Scientific Ltd, Vienna, Austria) were coated with $100 \mu$ of capture antibody. The plates were then sealed and incubated overnight at $4{ }^{\circ} \mathrm{C}$. This was followed by washing the plates thrice with washing buffer. After the last wash the plates were blocked with $100 \mu \mathrm{l}$ of blocking buffer and incubated for 2 hours at room temperature, then washed thrice using washing buffer. Specific standards for TNF- $a$, IFN- $y$, and IL-10 were diluted 2-fold with the $1 \mathrm{x}$ assay diluent. $100 \mu$ l of appropriate standard or $50 \mu \mathrm{l}$ of serum (probe) were added to separate wells followed by incubation for 2 hours at room temperature on a shaker. The plates were thereafter washed five times. The respective detecting antibodies were diluted with $1 x$ assay diluent then $100 \mu$ l of this added into each well followed by incubation for one hour at room temperature while shaking. Thereafter the plates were washed five times and then $100 \mu \mathrm{l}$ of Streptavidinperoxidase for IFN- $\gamma$ (1:200) and Avidin-HRP for IL-10 and TNF- $\alpha$ (1:200) were added into each well in appropriate plates followed by incubation for 30 minutes at room temperature. The wells were washed thrice using washing buffer. $100 \mu \mathrm{l}$ of TMB was added in each well then incubated for 15 minutes in the dark. Thereafter $50 \mu \mathrm{l}$ of the stop solution $\left(2 \mathrm{~N} \mathrm{H}_{2} \mathrm{SO}_{4}\right)$ was added to all the wells. The plates were then read using an ELISA reader at $450 \mathrm{~nm}$ wavelength.

\section{Post-mortem and Histological analysis}

At the end of the experiment, animals were humanely sacrificed and tissues harvested for histological analysis. Tissues from the small intestine (ileum) and caecum were processed, sectioned at $4 \mu \mathrm{m}$ using a rotex microtome (KD 2258, TED PELLA, Inc., California, USA), and subjected to hematoxylin-eosin staining followed by microscopic analysis.

\section{Statistical analysis}

Various statistical software programs were used for statistical analysis namely: R Statistics version 3.5.1 (The R Foundation, Vienna, Austria); SPSS v25 statistical software (IBM SPSS, New York, USA), and Graph Pad Prism 5.0 (Graph Pad Software, San Diego California, USA). ANOVA and Bonferroni post-hoc tests were used for comparison of the mean number of oocysts shedded and cytokine levels. Levels of significance were set at $95 \% \mathrm{Cl}$ with $\mathrm{p}<0.05$ considered as a significant difference. The correlation coefficient (R-values) was used to compare the relationship between the number of oocyst shedding and the lymphocytes being produced during the establishment of an infection. Probit analysis was performed for the prediction of minimum dosage capable of causing an infection using SPSS v25 statistical software (IBM SPSS, New York, USA).

\section{Results}




\section{a) Clinical parameters}

\section{i) Stool texture, body weight, and temperature changes}

Stool texture for experimental animals was either loose (stools spread on a surface), poorly formed (stools shape up when shed but easily spread to touch), or well-formed (hard stools that remain firm to touch), with a few animals showing watery stools (fluid stools that run on a surface). All control animals produced well-formed stools. This study showed that there were no significant differences in temperature as well as body weight changes between and within experimental groups and control groups $(p>0.05)$.

\section{ii) Oocysts shedding and infection doses}

In all experimental animals (oocysts doses: Group $1 ; 2 \times 10^{4}$, Group $2 ; 2 \times 10^{5}$, Group $3 ; 2 \times 10^{6}$ ) but not controls, the onset of oocysts shedding occurred between days 2 - 4 (mean $36.7 \pm 8.82,50.0 \pm 20.0$, 25.0 $\pm 5.0 ; p=0.56$; respectively; Figure 1) and continued through the experimental period with the highest oocysts shedding occurring between days $6-28$ consistently (mean 69.5 $59.3 ; 78.6 \pm 9.7 ; 73.2 \pm 6.7 ; p=0.82$, respectively). By day 30 , the number of oocysts shading averaged out to a constant of between 50 and 100 up till the end of the experiment. Thus, the experimental disease's acute phase occurred between days $6-28$, while the chronic phase begun from day 30 up to day 49 of the experiment.

\section{iii) Oocysts shedding patterns between males and females}

There were no significant differences between oocysts shedding patterns in males and females throughout the experimental period within and between infected groups $(p>0.05)$.

\section{b) Molecular typing of oocysts}

Molecular analysis (PCR followed by sequencing and alignment of the data) confirmed that oocysts used to infect the experimental animals in this study were those of Cryptosporidium parvum.

\section{c) Lymphocytes levels}

A Comparison of mean absolute lymphocytes between experimental animals and controls, especially during infection establishment (days 2-4), the acute phase (days 6-28) and the chronic phase (days $>30$ ), confirmed that lymphocytes increased in all experimental groups but not in the controls with a positive correlation between the lymphocytes and the number of oocyst shedding in day $0,7,14,21$ and 28 for all the experimental groups (Group 1; $r=0.82$; Group 2; $r=0.48$; Group 3; $r=0.43$ ).

\section{d) Immunological data (cytokines)}

Immunoassays revealed a significant increase in levels of pro-inflammatory cytokines (TNF-a, IFN- $\gamma$ ) and anti-inflammatory cytokine (IL-10) in experimental groups compared to controls on day 14 and 49 postinfection $(p<0.01$ ) (Figure $2 \mathrm{a}-\mathrm{c})$. This increase in inflammatory cytokine corresponded with an increase in the parasite dosage used for infection. It became evident that among the experimental groups, the levels 
of these cytokines were higher on day 49 compared to levels on day 14 post-infection (almost two folds). The observed significant increase in pro-inflammatory cytokines TNF- $\alpha$ and IFN- $\gamma$ corresponded with an upsurge in anti-inflammatory cytokine, IL-10 as parasite dosage increased in the experimental groups (Figure 2 a-c).

\section{e) Postmortem and Histopathological data}

Postmortem results showed signs of possible parasite infection in only experimental animals and not the controls. These included swollen mesenteric lymph nodes and spleen follicular hyperplasia; enlarged adrenal glands; blood vessels congestion in the colon, ileum, kidney, and brain; vanish gray hepatization with multiple focal fibrosis in the liver, a case of suspected hepatocystis. The possibility of the parasite (C. parvum) infection was indeed confirmed by histological data which revealed parasite establishment in the ileum and caecum epithelia in experimental animals but not in the controls (Figure 3 a-d).

\section{f) Prediction model for minimum infective dosage}

Using our data, the minimum hypothetical predictive infective dose (ID) for $50 \%\left(\right.$ ID $\left._{50}\right)$ required to cause an infection, was calculated through a Probit statistical model and found to be 25 oocysts.

\section{Discussion}

This study established a reproducible infant baboon model for childhood cryptosporidiosis studies using isolates of Cryptosporidium parvum. Various parameters supporting this model were evaluated including oocysts shedding, body weight and temperature changes, stool texture, lymphocytes, cytokines, postmortem observations, and histopathology. Herein, we compare our findings with those in humans, rhesus monkeys, and mice, which further confirms our model.

Clinical observation showed that stool texture for our experimental animals was either loose, poorly formed, or well-formed, with a few animals showing watery stools. All control animals produced wellformed stools. Similar observations exist in human and rhesus monkeys where diarrhea was an indication but did not necessarily predict infection [14]. These findings contrast those in mice, which do not show any clinical signs $[7,15]$.

In this study, there were no significant differences in temperature and body weight changes between and within experimental groups as observed in humans and rhesus monkey studies $[7,14]$.

In our study, all three different dosages established an infection indicating that a lower dosage of $2 \times 10^{4}$ oocysts was as effective as a higher dosage of $2 \times 10^{5}$ and $2 \times 10^{6}$. This low infective dosage was lower than the $2 \times 10^{5}$ reported in rhesus monkeys [7]. Though not done in this study, it is possible that an even lower dosage of $<2 \times 10^{4}$ oocysts could also have established infection. Indeed, the minimum hypothetical infective dose (ID) for $50 \%\left(\mathrm{ID}_{50}\right)$ required to cause an infection in a study, derived from a Probit 
statistical model, is 25 oocysts, which is closer to the 10-50 oocysts reported in rhesus monkeys [7] but far less than the $\mathrm{LD}_{50}$ of 79 oocysts for mice [15].

As to whether the risk of infection is gender-based, it was clearly demonstrated that oocysts shedding patterns in male and female baboons within and between infections groups were similar. This finding has been reported consistently in humans where the risk of contracting Cryptosporidium between sexes was insignificant [14] but such comparisons had not been demonstrated in rhesus monkeys [7].

The onset of oocysts shedding in all experimental animals but not controls between days $2-4$, with the highest oocyst shedding occurring between days $6-28$, considered as the acute phase of the disease in this study, is similar to observations in humans and rhesus monkey studies where infection establishes 3-7 days and oocyst shedding continues 3-4weeks independent of the dosage [7, 16-18]. However, this is in contrast to mice in which oocyst shedding occurs on day 6 post-inoculation, and lasts for only 5-12 days $[5,19]$.

As expected, experimental animals and not the controls showed oocysts embedding onto the epithelial mucosa of both the caecum and ileum as demonstrated in other studies, in mice and humans, where oocysts were observed to embed onto the epithelial lining of the duodenum, jejunum and ileum $[20,21]$.

Analysis of lymphocytes indicated a positive correlation with the number of oocyst shedding as shown in days $0,7,14,21,28$ for all the experimental groups. The increase in the number of lymphocytes relative to the increase in shedded oocysts indicated a successful Cryptosporidium infection. This is similar to humans but has not been evaluated in rhesus monkeys and mice $[16,17]$.

The pro-inflammatory cytokines, TNF- $\alpha$ and IFN- $\gamma$, are good indicators of the establishment of parasite infection in most apicomplexan protozoan infections, similar to what would be observed in Plasmodium spp in malaria and Toxoplasma gondii in toxoplasmosis [22]. The observed significant increase in proinflammatory cytokines TNF-a and IFN- $y$ in our current study suggests a Th1 immune response at play. This up-regulation of TNF- $a$ and IFN- $\gamma$ can be interpreted as either enhancing protection against Cryptosporidium or rather causing tissue inflammation and is corroborated by tissue pathology from histology sections, which demonstrated the presence of parasite on the epithelium of both ileum and caecum of only experimental animals but not the controls. The observed rise in IL-10 levels is indicative of the body's attempt at regulating the pro-inflammatory cytokines to bring about the balance of interplay between innate and adaptive immune response. Furthermore, elevation in levels of cytokines (TNF-a, IFN$\gamma$, and IL-10) in experimental animals but not in the control animals, corresponds to a study on humans [23] and also corroborates the histological analyses, confirming the establishment of Cryptosporidium infection in the experimental animals and not the controls. Indeed, the increase in these cytokines also corresponded with an increase in the number of Cryptosporidium oocysts shedding in the experimental animals.

Besides, the pathologies observed in experimental animals during postmortem results in our study was an indication of tissue inflammation/swelling, which may partly be explained by immune reactions 
elicited by the infection $[24,25]$ as well as possible modulated immunity due to gut microbiome imbalance possibly resulting from the presence of Cryptosporidium in the gut. All these may have contributed to a cytokine storm elicited by the infection, leading to the observed global organ pathologies [26].

\section{Conclusions}

The series of experiments conducted in this study resulted in the establishment of a reproducible infant baboon infection model with an effective minimum infection dose of $2 \times 10^{4}$ oocysts of Cryptosporidium parvum. This experimental model is a useful tool for studying the pathogenesis of childhood cryptosporidiosis.

\section{Abbreviations}

PBS: Phosphate buffered saline

DNA: Deoxyribonucleic acid

PCR: Polymerase chain reaction

ASL: Adenylosuccinate lyase

RNA: Ribonucleic acid

NCBI: National center for biotechnology information

UV: Ultraviolet

bp: base pairs

Co.: Company

Ltd: Limited

ELISA: Enzyme-linked immunosorbent assay

TMB: Tetramethylbenzidine

ANOVA: Analysis of variance

SPSS: statistical package for the social sciences

TNF-a: Tumor necrosis factor-alpha

IFN-ץ: Interferon gamma 


\section{Declarations}

\section{Ethics approval}

The experimental procedures were reviewed and passed by the Institute of Primate Research Scientific and Ethics Review Committee (ISERC) and the Institutional Animal Care and Use Committee (IACUC) for scientific soundness and animals' welfare issues before commencement of the study (ISERC/13/16).

\section{Consent for publication}

Not Applicable

\section{Availability of Data and materials}

The datasets used and/or analyzed during the current study are available from the corresponding author on reasonable request.

\section{Competing interests}

The authors declare that that they have no competing interests.

\section{Funding}

This project was supported by a Grand challenges exploration grant from Bill and Melinda Gates Foundation [Opportunity ID OPP1160347].

\section{Authors' contributions}

NEJ: Was involved in concept development, all experimental designs and execution, data analysis and interpretation, and played a major role in writing the manuscript.

AN: Was involved in concept development, all experimental designs and execution, data analysis and interpretation, and played a major role in writing the manuscript.

DCC: Was involved in concept development, all experimental designs and execution, histology data analysis and interpretation, and played a role in the manuscript writing.

JNN: Was involved in immunological experimental design and execution, data analysis and interpretation, and was involved in the manuscript writing.

\section{Acknowledgements}

We thank Kenneth Waititu, Gift Katana, and Samson Mutura for the technical assistance. We also thank Abel Haro for statistical analysis. 


\section{References}

1. Leitch GJ, He Q. Cryptosporidiosis-An Overview. Journal of Biomedical Research 2011; 25: 1-16

2. Shirley Debbie-Ann T, Moonah SN, Kotloff KL. Burden of disease from Cryptosporidiosis. Curr Opin Infect Dis 2012;25:555-563

3. Baamaiyi PH, Redhuan NEM. Prevalence and risk factors for cryptoaporidiosis: a global, emerging, neglected zoonosis. Asian Biomedicine 2016;10:309-325

4. Current WL, Garcia LS. Cryptosporidiosis. Clin Microbiol Rev 1991;4:325-358

5. Sharma P, Sharma A., Sehgal R, Malla N, Khurana S. Genetic diversity of Cryptosporidium isolates from patients in North India. International Journal of Infectious Diseases 2013;17: e601-e605

6. Opintan JA, Newman MJ, Ayeh-Kumi PF et al. Pediatric diarrhea in southern Ghana: etiology and association with intestinal inflammation and malnutrition. Am J Trop Med Hyg 2010;83:936-943

7. Miller RA; Bronsdon MA, Morton WR. Experimental Cryptosporidiosis in a Primate Model. Journal of Infectious Diseases 1990;161:312-315

8. Siwila J, Phiri IG, Enemark HL, Nchito M, Olsen A. Intestinal helminths and protozoa in children in preschools in Kafue district, Zambia. Trans R Soc Trop Med Hyg 2010;104:122-128

9. Chappell CL, Okhuysen PC, Sterling CR, DuPont HL. Cryptosporidiumparvum: intensity of infection and oocyst excretion patterns in healthy volunteers. J Infect Dis. 1996;173:232-236

10. Jokipii L, Jokipii AM. Timing of symptoms and oocyst excretion in human cryptosporidiosis. N Engl J Med 1986;315:1643-1647

11. Kváč M, Kestránová M, Pinková M et al. Cryptosporidium scrofarum n. sp. (Apicomplexa: Cryptosporidiidae) in domestic pigs (Sus scrofa). Vet Parasitol 2013;191:218-27

12. Li W, Kiulia NM, Mwenda JM et al. Cyclospora papionis, Cryptosporidium hominis, and HumanPathogenic Enterocytozoon bieneusi in Captive Baboons in Kenya. Journal of Clinical Microbiology 2011;49:4326-4329

13. Bairami KA, Rezaeian M, Zeraati H et al. A Sensitive and Specific PCR Based Method for Identification of Cryptosporidium Sp. Using New Primers from 18S Ribosomal RNA. Iran J Parasitol 2011; 6:4. 5/18/2021 Iranian Journal of Parasitology https://ijpa.tums.ac.ir/index.php/ijpa/article/view/198

14. Parsons MB, Travis D, Lonsdorf EV et al. Epidemiology and Molecular Characterization of Cryptosporidium spp in Humans, Wild Primates, and Domesticated Animals in the Greater Gombe Ecosystem, Tanzania. PLoS Negl Trop Dis 2015;10:e0003529

15. Finch GR, Daniels CW, Black EK, Schaefer III FW, Belosevic M. Dose Response of Cryptosporidium parvam in Outbred Neonatal CD-1 Mice. Applied and Environmental Microbiology 1993;59:3661-3665

16. DuPont HL, Chappell CL, Sterling CR, Okhuysen PC, Rose JB, Jakubowski W. The infectivity of Cryptosporidium in healthy volunteers. The New England Journal of Medicine 1995;332:855-859 
17. Chappel C, Okhuysen PC, Langer-Curry RC et al. Cryptosporidiummeleagridis: infectivity in healthy adult volunteers. Am J Trop Med Hyg 2011;85:238-242

18. Okhuysen PC, Chappell CL, Sterling CR, Jakubowski W, DuPont HL. Susceptibility and Serologic Response of Healthy Adults to Reinfection with Cryptosporidium parvam. Infection and Immunology 1998;66:441-443

19. Matsuo T, Tsuge $Y$, Umemiya-Shirafuji R, Fujino T, Matsui T. Adaptation and Immunogenecity of Cryptosporidium parvum to immunocompetent mice. Acta Parasitologica 2014;59:189-192

20. Hemphil A, Muller N, Muller J. Comparative pathobiology of the Intestinal protozoan parasites Giardia lamblia, Entamoeba hystolytica and Cryptosporidium parvum. Pathogens 2019; 8:1-22

21. Al-Warid HS, Al-Saqur IM, Mahmood SH. Histopathological changes in mice infected with Cryptosporidium spp. Int Jour of Pharm and Bio Sci 2013;3:3. 5/18/2021 International Journal of Pharmacy and Biological Sciences/Research https://www.ijpbs.com/ijpbsadmin/upload/ijpbs_5225c57b328dd.pdf

22. Nyariki JN, Mokaya D, Jillani NE, Nyamweya NO, Orina Al. Oral Administration of Coenzyme Q10 has the Capacity to Stimulate Innate Lymphoid Cells Class Two during Experimental Cerebral Malaria. South Asian Journal of Parasitology 2019;2:2. 5/18/2021 South Asian Journal of Parasitology/Research https://journalsajp.com/index.php/SAJP/article/view/29966

23. Pantenburg B, Dann SM, Wang Heuy-Ching et al. Intestinal Immune Response to Human Cryptosporidium sp infection. Infection and Immunity 2008;76:23-29

24. AboueL-Nour MF, El-Shewehy DMM, Hamada SF, Morsy TA. The efficacy of three medicinal plants: Garlic, Ginger and Mirazid and a chemical drug Metronidazole against Cryptosporidium parvum.1immunological response. J Egypt Soc Parasito/ 2015;45(3):559 -570

25. Lean IS, McDonald V, Pollok RC. The role of cytokines in the pathogenesis of Cryptosporidium infection. Curr Opin Infect Dis 2002;15(3):229-34

26. Tisoncik JR, Korth MJ, Simmons CP, Farrar J, Martin TR, Katze MG. Into the Eye of the Cytokine Storm. Microbiology and Molecular Biology Reviews, 2012;76(1): 16-32

\section{Figures}




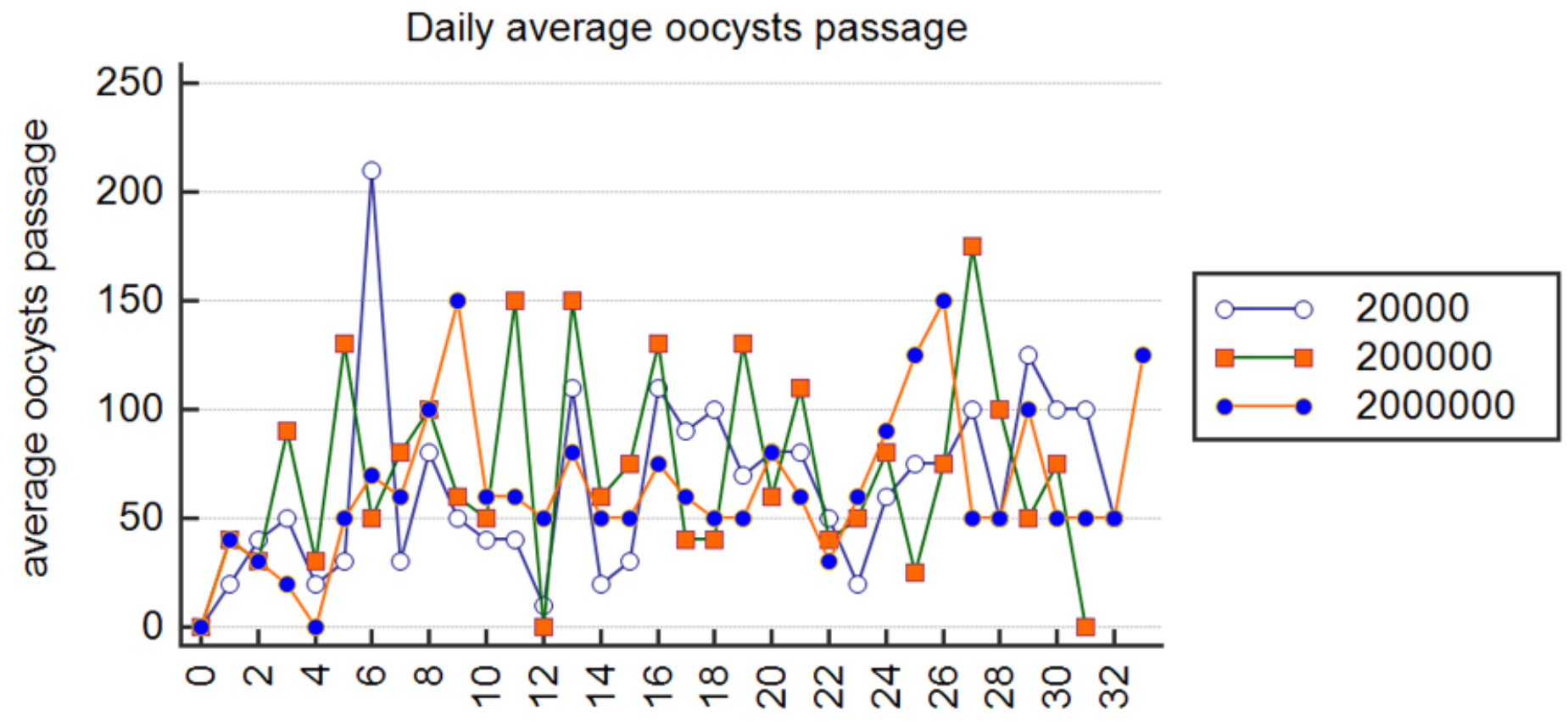

\section{Day}

Figure 1

Plots of daily average oocyst shading for the different doses using R Statistics version 3.5.1. In all experimental animals, the onset of oocysts shedding occurred between days 2 and 4 and continued through the experimental period with the highest oocysts shedding occurring between days 6 and 28 indicating that the experimental disease's acute phase occurred between days 6 and 28, while the chronic phase begun from day 30 up to day the end of the experiment.

\begin{tabular}{|l|l|l|l|l|}
\hline & & \\
\hline
\end{tabular}




\section{Figure 2}

Comparison of levels of pro-inflammatory and anti-inflammatory cytokines between experimental and control animals (a-c): Showing levels of pro-inflammatory cytokines (TNF-a, IFN- $\gamma$ ) and anti-inflammatory cytokine (IL-10) in experimental groups compared to controls on day 14 and 49 post infection using Graph Pad Prism 5.0. a) pro-inflammatory cytokine TNF-alpha increased (almost two folds) in day 14 as compared to day 49 in the experimental groups as parasite dosage increased. b) pro-inflammatory cytokine IFN gamma increased (almost two folds) in day 14 as compared to day 49 in the experimental groups as parasite dosage increased. c) anti-inflammatory cytokine IL-10 increased (almost two folds) in day 14 as compared to day 49 in the experimental groups as parasite dosage increased.

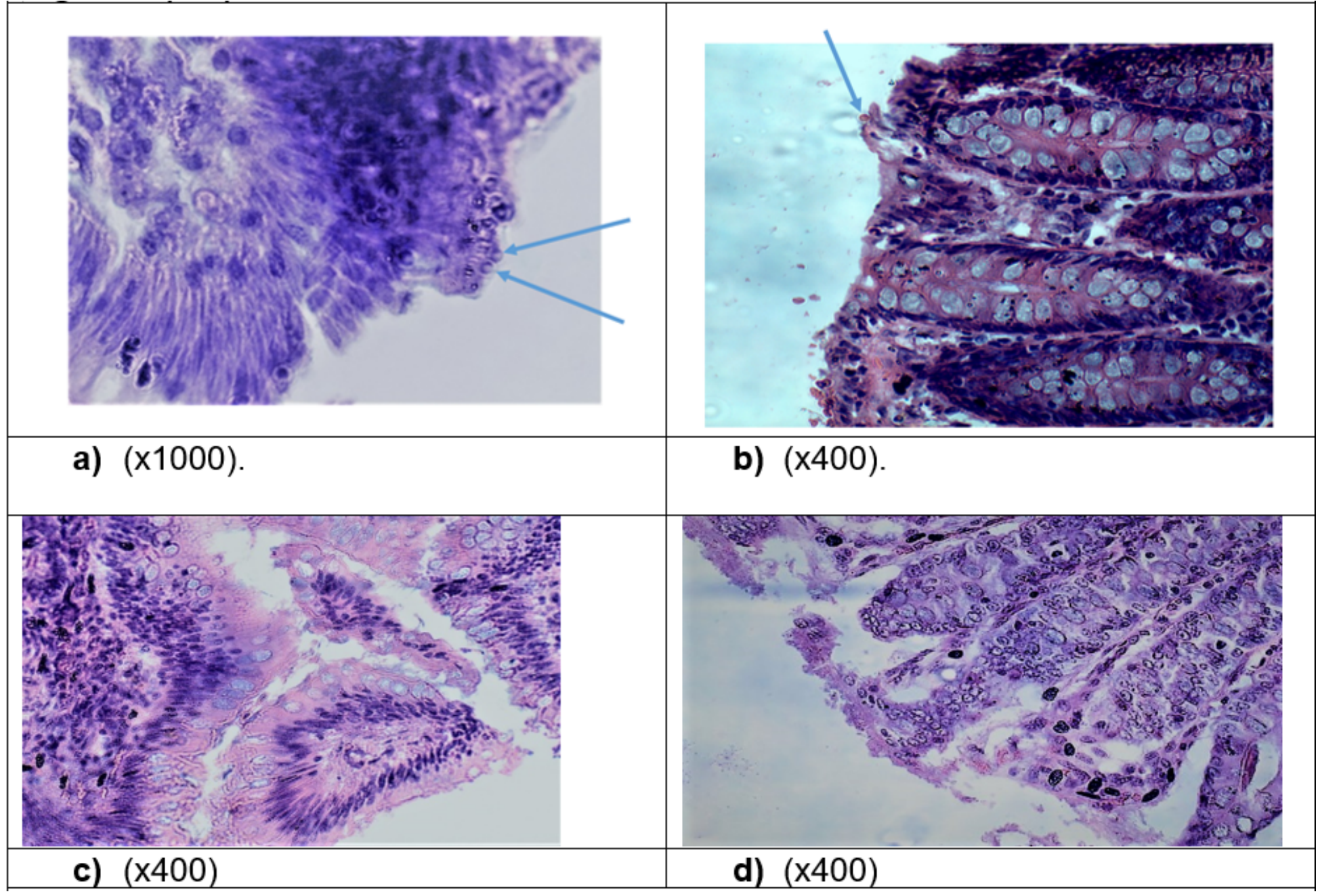

\section{Figure 3}

Comparison of parasite establishment within ileum and caecum epithelia of experimental and control animals (a-d): Demonstrates parasite establishment in a vacuole within the epithelia of ileum and caecum in only experimental animals and not the controls. Both a) and b) show the establishment of the parasite in the epithelial mucosa of the ileum and caecum in experimental animals, while c) and d) (controls) show normal ileum and caecum mucosa without parasite infection. a) Photomicrograph of ileum section with oocysts embedded in mucosa lining (x1000). b) Photomicrograph of caecum section 
with oocysts embedded in mucosa lining (x400). c) Photomicrograph showing no oocysts in mucosa of normal ileum epithelia in controls (x400). d) Photomicrograph showing no oocysts in mucosa of normal caecum epithelia in controls (x400).

\section{Supplementary Files}

This is a list of supplementary files associated with this preprint. Click to download.

- Baboon.jpg 